\title{
Orexin receptor antagonists reverse aberrant dopamine neuron activity and related behaviors in a rodent model of stress-induced psychosis
}

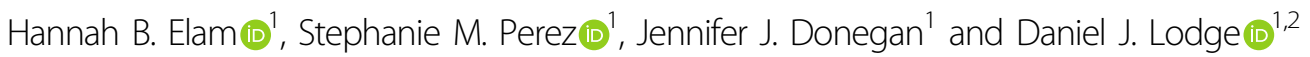

\begin{abstract}
Post-traumatic stress disorder (PTSD) is a prevalent condition affecting approximately $8 \%$ of the United States population and $20 \%$ of United States combat veterans. In addition to core symptoms of the disorder, up to $64 \%$ of individuals diagnosed with PTSD experience comorbid psychosis. Previous research has demonstrated a positive correlation between symptoms of psychosis and increases in dopamine transmission. We have recently demonstrated projections from the paraventricular nucleus of the thalamus (PVT) to the nucleus accumbens (NAc) can regulate dopamine neuron activity in the ventral tegmental area (VTA). Specifically, inactivation of the PVT leads to a reversal of aberrant dopamine system function and psychosis-like behavior. The PVT receives dense innervation from orexin containing neurons, therefore, targeting orexin receptors may be a novel approach to restore dopamine neuron activity and alleviate PTSD-associated psychosis. In this study, we induced stress-related pathophysiology in male Sprague Dawley rats using an inescapable foot-shock procedure. We observed a significant increase in VTA dopamine neuron population activity, deficits in sensorimotor gating, and hyperresponsivity to psychomotor stimulants. Administration of selective orexin 1 receptor $\left(O X_{1} R\right)$ and orexin 2 receptor $\left(\mathrm{OX}_{2} \mathrm{R}\right)$ antagonists (SB334867 and EMPA, respectively) or the FDA-approved, dual-orexin receptor antagonist, Suvorexant, were found to reverse stress-induced increases in dopamine neuron population activity. However, only Suvorexant and SB334867 were able to reverse deficits in behavioral corelates of psychosis. These results suggest that the orexin system may be a novel pharmacological target for the treatment of comorbid psychosis related to PTSD.
\end{abstract}

\section{Introduction}

Post-traumatic stress disorder (PTSD) is a psychiatric disorder that develops following exposure to a traumatic event and is characterized by flashbacks to the traumatic event, avoidance of event-related stimuli, hypervigilance, and cognitive deficits ${ }^{1}$. In addition, $80-90 \%$ of individuals diagnosed with PTSD receive a comorbid psychiatric diagnosis, including depression, anxiety, and psychosis ${ }^{2-5}$. While PTSD and comorbid depression and anxiety have been extensively studied, less is known about psychosis in

\footnotetext{
Correspondence: Hannah B. Elam (elamh@uthscsa.edu)

${ }^{1}$ Department of Pharmacology and Center for Biomedical Neuroscience,

University of Texas Health Science Center, San Antonio, TX 78229, USA

${ }^{2}$ South Texas Veterans Health Care System, Audie L. Murphy Division, San

Antonio, USA
}

PTSD patients, despite the fact that up to $64 \%$ of individuals with PTSD are diagnosed with comorbid psychosis $^{5,6}$. Antipsychotic medications are commonly prescribed and effectively lessen symptoms of psychosis, however, they produce many unwanted side effects that often result in poor patient compliance ${ }^{7}$. Although it has been repeatedly demonstrated that hyperactivity in the dopamine system contributes to symptoms of psychosis, targeting the dopamine system directly results in unwanted side effects. Interestingly, no obvious histopathology has been identified within the mesolimbic dopamine neurons of patients with psychosis ${ }^{8,9}$. Rather, the pathology appears to lie in upstream brain regions that regulate dopamine neuron activity ${ }^{10,11}$. One such region of interest, known to regulate dopamine system function, is the

\section{(c) The Author(s) 2021}

(c) (i) Open Access This article is licensed under a Creative Commons Attribution 4.0 International License, which permits use, sharing, adaptation, distribution and reproduction cc) in any medium or format, as long as you give appropriate credit to the original author(s) and the source, provide a link to the Creative Commons license, and indicate if changes were made. The images or other third party material in this article are included in the article's Creative Commons license, unless indicated otherwise in a credit line to the material. If material is not included in the article's Creative Commons license and your intended use is not permitted by statutory regulation or exceeds the permitted use, you will need to obtain permission directly from the copyright holder. To view a copy of this license, visit http://creativecommons.org/licenses/by/4.0/. 
paraventricular nucleus of the thalamus (PVT). We have previously demonstrated that chemogenetic activation of neurons projecting from the PVT to the nucleus accumbens (NAc) produces a significant increase in ventral tegmental area (VTA) dopamine neuron population activity, defined as the number of neurons firing spontaneously ${ }^{11}$. Conversely, inhibition of this pathway, in a rodent model used to study the physiological and behavioral correlates of psychosis, can reverse aberrant VTA dopamine neuron activity ${ }^{12}$. Additionally, the PVT has been shown to be hyperactive following stress, suggesting increased signaling from the PVT to the NAc, following stress, may contribute to symptoms of psychosis in individuals with $\mathrm{PTSD}^{13}$.

Orexin-containing neurons, originate in the lateral hypothalamus and densely innervate the PVT, making them an ideal target to modulate the activity of the $\mathrm{PVT}^{14,15}$. The orexin system consists of two neuropeptides cleaved from a single precursor protein, orexin peptide A (OXA) and orexin peptide B (OXB) which bind to and activate two distinct Gq-protein-coupled receptors, orexin 1 receptor $\left(\mathrm{OX}_{1} \mathrm{R}\right)$ and orexin 2 receptor $\left(\mathrm{OX}_{2} \mathrm{R}\right)$. $\mathrm{OX}_{1} \mathrm{R}$ binds $\mathrm{OXA}$ with high affinity and binds $\mathrm{OXB}$ with much lower affinity while $\mathrm{OX}_{2} \mathrm{R}$ has been shown to have equal affinity for both OXA and $\mathrm{OXB}^{16}$. Further, both orexin receptors are highly expressed within the $\mathrm{PVT}^{17,18}$. The orexin system was first discovered as a modulator of sleep and appetite, but recent studies suggest this neuropeptide system plays a pleiotropic role, regulating a variety of biological processes, such as pain, cardiovascular function, and neuroendocrine regulation ${ }^{19-22}$. Although the orexin system is widespread and diverse in function, recent studies have shown that targeting this system may be beneficial in treating substance use disorder as well as psychosis $^{22-25}$. Importantly, both OXA and OXB have been shown to dose-dependently increase the firing rate of PVT neurons and increase dopamine neuron population activity, without affecting the firing rate of these neurons in vivo ${ }^{26,27}$.

We have recent data demonstrating the ability of the dual-orexin receptor antagonist (DORA), TCS1102, to reverse aberrant dopamine neuron population activity in a rodent model used to examine psychosis-like behaviors, when administered both systemically and intracranially into the $\mathrm{PVT}^{27}$. However, little is known about the ability of the FDA-approved DORA, Suvorexant, to reverse psychosis-like deficits in a rodent model displaying stressinduced pathophysiology relevant to $\mathrm{PTSD}^{28-30}$. Here, we used a two-day inescapable foot-shock paradigm to induce robust increases in dopamine neuron population activity within the VTA. Additionally, we observed deficits in two dopamine-dependent behavioral correlates of psychosis, pre-pulse inhibition of startle (PPI) and hyperresponsivity to psychomotor stimulants $(\mathrm{MK}-801)^{31-33}$. We then investigated the ability of pharmacological inhibition of orexin receptor activity to reverse both increases in VTA dopamine neuron activity and deficits in dopaminedependent behaviors. We found that of the FDA-approved DORA, Suvorexant, was able to reverse aberrant dopamine transmission in the VTA following two-day inescapable foot-shock stress. Additionally, Suvorexant attenuated stress-induced behavioral deficits in PPI and MK-801 induced locomotor activity. Furthermore, we found that systemic administration of either the selective $\mathrm{OX}_{1} \mathrm{R}$ antagonist, SB334867 or the selective $\mathrm{OX}_{2} \mathrm{R}$ antagonist, EMPA, was able to reverse increased VTA dopamine neuron activity following stress. However, deficits in PPI were only reversed following administration of SB334867, not EMPA. These findings suggest that targeting the orexin system, specifically the $\mathrm{OX}_{1} \mathrm{R}$, can reverse aberrant dopamine neuron activity that contributes to psychosislike behavior and that Suvorexant, or an $\mathrm{OX}_{1} \mathrm{R}$ antagonist, may be a novel therapeutic intervention for the treatment of comorbid psychosis in PTSD.

\section{Materials and methods}

All experiments were performed in accordance with the guidelines outlined in the USPH Guide for the Care and Use of Laboratory Animals and were approved by the Institutional Animal Care and the Use Committees of UT Health San Antonio and U.S. Department of Veterans Affairs.

\section{Animals}

Adult male Sprague Dawley rats (250-275 g) were obtained from Envigo RMS Inc. (Indianapolis, IN, USA) and used for all experiments. Rats were maintained in a temperature-controlled environment, on a $12 \mathrm{~h} / 12 \mathrm{~h}$ light/dark cycle, with ad libitum access to food and water. Animals were randomly allocated to various experimental groups. Experimenters were blinded to treatment groups for all behavioral tests.

\section{Drug administration}

Suvorexant, the highly selective dual-orexin antagonist, $(10 \mathrm{mg} / \mathrm{kg}$ or $30 \mathrm{mg} / \mathrm{kg}$; OX1R, $\mathrm{Ki}=0.54 \mathrm{nM}$; OX2R, $\mathrm{Ki}=$ $0.57 \mathrm{nM}^{34}$ ) or vehicle (DMSO) were administered intraperitoneally $30 \mathrm{~min}$ prior to electrophysiological recordings or behavioral assays. For experiments examining selective orexin receptor activity, the selective $\mathrm{OX}_{1} \mathrm{R}$ antagonist, SB334867 $\left(10 \mathrm{mg} / \mathrm{kg} ; \mathrm{OX}_{1} \mathrm{R}, \mathrm{Ki}=99 \mathrm{nM}\right.$; $\mathrm{OX}_{2} \mathrm{R} \mathrm{Ki}=>10,000 \mathrm{nM}^{35}$ ) the selective $\mathrm{OX}_{2} \mathrm{R}$ antagonist, EMPA, $\left(10 \mathrm{mg} / \mathrm{kg} ; \mathrm{OX}_{1} \mathrm{R}, \mathrm{Ki}=900 \mathrm{nM} ; \mathrm{OX}_{2} \mathrm{R} \mathrm{Ki}=\right.$ $\left.1.45 \mathrm{nM}^{36}\right)$, or vehicle (10\% DMSO and $10 \% \quad 2$ hydroxypropyl-b-cyclodextrin in sterile water) were given 20 min prior to electrophysiological recordings or behavioral assays. 


\section{Two-day inescapable foot-shock}

Rats were placed in a $30.5 \times 25.4 \times 30.5 \mathrm{~cm}^{3}$ square conditioning chamber with metal walls and a stainlesssteel grid shock floor (Coulbourn Instruments, Whitehall, PA, USA). Rats randomly assigned to the stress group received a two-day inescapable shock treatment, where each day they received $60 \times 15 \mathrm{~s} 0.8 \mathrm{~mA}$ foot shocks with an inter-trial interval (ITI) of $30 \mathrm{~s}$ with a $25 \%$ deviation $(+/-7.5 \mathrm{~s})$. Control rats were handled daily but not exposed to conditioning chambers. Electrophysiological experiments and PPI assays were conducted $24 \mathrm{~h}$ following inescapable stress. MK-801 induced locomotor response assays were conducted $48 \mathrm{~h}$ following inescapable stress.

\section{In vivo extracellular dopamine neuron recordings}

Rats were anesthetized with $8 \%$ chloral hydrate $(400 \mathrm{mg} / \mathrm{kg}$, i.p.) and placed in a stereotaxic apparatus. Chloral hydrate was used for all dopamine recordings to avoid significantly depressing dopamine neuron activity ${ }^{37}$. Supplemental anesthesia was administered to maintain suppression of limb compression withdrawal reflex. Core body temperature of $37^{\circ} \mathrm{C}$ was sustained using a thermostatically controlled heating pad (PhysioSuite, Kent Scientific Coorporation, Torrington, CT, USA). Extracellular glass microelectrodes (impedance $\sim 6-10 \mathrm{M} \Omega$ ) were lowered into the VTA (posterior 5.3 to 5.7, lateral 0.6 to 1.0 from bregma, and -6.5 to $-9.0 \mathrm{~mm}$ ventral of the brain surface) using a hydraulic micro-positioner (Model 640, Kopf Instruments). Spontaneously active dopamine neurons were recorded using previously established electrophysiological criteria $^{38,39}$. Open filter settings (low-frequency cutoff: $30 \mathrm{~Hz}$; high-frequency cutoff: $30 \mathrm{kHz}$ ) were used to identify and record dopamine neurons in the VTA. Various regions of the VTA were sampled by making multiple 6-9 vertical passes, separated by $200 \mu \mathrm{m}$, in a predetermined pattern. Three parameters of dopamine activity were measured and analyzed: the number of dopamine neurons firing spontaneously (population activity) ${ }^{11}$, basal firing rate, and proportion of action potentials occurring in bursts. Electrophysiological analysis of dopamine neuron activity was performed using commercially available computer software (LabChart version 8; ADInstruments, Colorado Springs, CO, USA) and analyzed with Prism software (GraphPad Software, San Diego, CA, USA). All orexin antagonists were administered thirty minutes prior to extracellular recordings and single-cell extracellular recordings lasted no longer than two hours post injection.

\section{Pre-pulse inhibition of startle response (PPI)}

Rats were placed in a sound attenuated chamber (SD Instruments, San Diego, CA, USA) and allowed to acclimate to $65 \mathrm{~dB}$ background noise for $5 \mathrm{~min}$. Rats were then exposed to ten startle-only trials $(40 \mathrm{~ms}, 120 \mathrm{~dB}, 15 \mathrm{~s}$ average inter-trial intervals (ITI)). Next, rats were exposed to 24 trials where a pre-pulse $(20 \mathrm{~ms}$ at $69 \mathrm{~dB}, 73 \mathrm{~dB}$ and $81 \mathrm{~dB}$ ) was presented $100 \mathrm{~ms}$ before the startle pulse. Each pre-pulse + startle pulse and startle pulse were presented 6 times in a pseudo-random order ( $15 \mathrm{~s}$ average ITI). The startle response was measured from $10-80 \mathrm{~ms}$ after the onset of the startle only pulse and recorded using SR-LAB Analysis Software (SD Instruments). All orexin antagonists were administered thirty minutes prior behavioral assays.

\section{MK-801 induced locomotor response}

Rats were placed in an open field arena (Med Associates, VT, USA) and spontaneous locomotor activity in the $x-y$ plane was determined for $30 \mathrm{~min}$ by beam breaks and recorded with Open Field Activity software (Med Associates). Following a 30-min baseline recording, all rats were injected with MK-801 (75 $\mu \mathrm{g} / \mathrm{kg}$, i.p.). Locomotor activity was recorded for an additional $30 \mathrm{~min}$ immediately following dose.

\section{Histology}

Immediately following all electrophysiological recordings, rats were rapidly decapitated. Brains were post-fixed for at least $24 \mathrm{~h}$ ( $4 \%$ formaldehyde in saline) and cryoprotected $(25 \% \mathrm{w} / \mathrm{v}$ sucrose in PBS) until saturated. Coronal sections $(25 \mu \mathrm{m})$ were collected on a cryostat (Leica) and mounted onto gelatin-chrom-coated slides and stained with neutral red $(0.1 \%)$ and thionin acetate $(0.01 \%)$ for histological verification of electrode tracks within the VTA ${ }^{40}$.

\section{Statistical analysis}

Electrophysiological data were analyzed by two-way ANOVA (stress $x$ drug). Holm-Sidak was used for all post hoc analysis, with significance determined at $\mathrm{p}<0.05$. PPI and MK-801 induced locomotor activity were analyzed using SigmaPlot (Systat Software Inc., Chicago, IL, USA). PPI Data were analyzed by three-way ANOVA and the Holm-Sidak post hoc test, with significance determined at $p<0.05$. Locomotor data were analyzed by two separate three-way ANOVA's (stress $\times$ drug $\times$ time), one for each of the relevant treatments (baseline, MK-801) followed by a Holm-Sidak post hoc test. All data are represented as the mean \pm SEM, unless otherwise stated, with $n$ values representing the number of rats per group unless otherwise specified. All data sets were checked for normality and variance was found to be similar between experimental groups. Sample sizes were determined based on our previous studies ${ }^{41,42}$.

\section{Materials}

Suvorexant (Item No. 9002140; Cayman Chemical, Ann Arbor, MI, USA) was made fresh daily and dissolved in dimethylsulfoxide (DMSO). SB334867 and EMPA (Cat. No 1960 and 4558; Tocris, Minneapolis, MN, USA) were 
made fresh daily and dissolved in 10\% DMSO and 10\% 2hydroxypropyl-b-cyclodextrin (Item No. 16169; Cayman Chemical, Ann Arbor, MI, USA) in sterile water. Chloral hydrate and DMSO were purchased from Sigma-Aldrich (St. Louis, MO, USA). All other chemicals and reagents were of either analytical or laboratory grade and purchased from standard suppliers.

\section{Results}

Suvorexant, a dual-orexin antagonist, reverses aberrant VTA dopamine neuron population activity

The dopamine hypothesis, one of the longest-standing hypotheses of psychosis, suggests an increase in dopamine activity correlates with severity of psychotic symptoms ${ }^{8,9}$. In rats, changes in mesolimbic dopamine activity can be examined using in vivo extracellular electrophysiology to quantify the number of spontaneously active VTA dopamine neurons ${ }^{10,38,39}$. Following two-day inescapable shock, we found a main effect of stress on VTA dopamine neuron population activity (Fig. 1; two-way ANOVA; factors: stress $\times$ drug; $F_{(1,35)}=$ $7.731 ; t=2.781 ; P=0.009, n=6$ /group). Stressed animals treated with vehicle displayed significantly higher number of spontaneously active dopaminergic cells per track when compared to control, vehicle treated animals (Holm-Sidak; $t=4.033, p=<0.001, n=6$ /group; control/vehicle: $n=6$ rats; $1.11 \pm 0.09$ cells per track; stress/ vehicle: $n=6$ rats; $1.86 \pm 0.24$ cells per track). This stress-induced increase in dopamine neuron activity was not observed in rats treated with either 10 or $30 \mathrm{mg} / \mathrm{kg}$ of Suvorexant and was completely reversed by the $30 \mathrm{mg} / \mathrm{kg}$ dose $(p=0.005, n=6$ rats; $1.22 \pm 0.09$ cells per track). No significant differences were observed in the firing rate (Stress/vehicle: $n=61$ neurons; $4.17 \pm$ $0.25 \mathrm{~Hz}$; stress $/ 10 \mathrm{mg}: n=49$ neurons; $3.69 \pm 0.25 \mathrm{~Hz}$; control/30 mg: $n=51$ neurons; $3.97 \pm 0.25 \mathrm{~Hz}$; stress/ $30 \mathrm{mg}: n=41$ neurons; $4.11 \pm 0.38 \mathrm{~Hz})$ or percentage bursting in any of the groups (control/vehicle: $n=40$ neurons; $33.76 \pm 4.35 \%$; stress/vehicle: $n=61$ neurons; $32.01 \pm 3.48 \%$; control/10 mg: $n=37$ neurons; $26.26 \pm$ 4.03\%; stress/10 mg: $n=49$ neurons; $28.33 \pm 3.41 \%$; control/30 mg: $n=51$ neurons; $27.16 \pm 3.49 \%$; stress/ 30 mg: $n=41$ neurons; $27.69 \pm 4.36 \%$ ).

\section{Suvorexant reverses stress-induced deficits in PPI}

Individuals with psychosis typically display deficits in sensorimotor gating, which can be measured in both humans and animal models of the disease using pre-pulse inhibition (PPI) ${ }^{31}$. Initial pilot studies demonstrated that the high dose of Suvorexant $(30 \mathrm{mg} / \mathrm{kg})$ dramatically reduced spontaneous locomotor activity (by $>80 \%$ ) whereas the lower dose of $10 \mathrm{mg} / \mathrm{kg}$ did not (Fig. 2). For this reason, $10 \mathrm{mg} / \mathrm{kg}$ was used for the following behavioral studies. Consistent with previous literature, we found a main effect of pre-pulse intensity, demonstrated by an attenuated startle response as the pre-pulse magnitude increases (Fig. 2; $P<0.001 ; n=16$ rats/group). Additionally, a main effect of stress was observed (threeway ANOVA; factors: stress $\mathrm{x}$ drug $\mathrm{x}$ pre-pulse intensity; $\left.F_{(1,191)}=5.845 ; t=2.418 ; P=0.017\right)$ demonstrating stress-induced deficits in PPI at all pre-pulse intensities. Stress/vehicle rats displayed significant deficits in PPI (Fig. 2; Holm-Sidak: $t=3.167, p=0.002$ ) when compared to control/vehicle. Importantly, PPI deficits observed in stress/vehicle animals were significantly reversed by Suvorexant $(10 \mathrm{mg} / \mathrm{kg}) \quad$ (Holm-Sidak: $t=2.014, \quad p=$ 0.046). No differences were observed between vehicle and Suvorexant $(10 \mathrm{mg} / \mathrm{kg})$ in unstressed controls (Holm-Sidak: $t=0.902, p=0.368$ ). The average startle response of Suvorexant treated animals was not significantly different from control (two-way ANOVA; factors: stress $\times$ drug; $\left.F_{(1,63)}=0.593 ; t=0.770 ; P=0.444\right)$. These results suggest repeated foot-shock stress can induce deficits in sensorimotor gating, as measured by PPI, and that these deficits can be alleviated by treatment with Suvorexant.

\section{MK-801 induced locomotor activity increases after stress but is reversed following administration of Suvorexant}

Patients with psychosis display hyper-sensitivity to psychomotor stimulants which can be modeled in rats by measuring locomotor activity before and after administration of the NMDA antagonist, MK- $801^{10,41,43}$. Consistent with previous literature ${ }^{44}$, stress/vehicle rats showed an increase in locomotor activity at baseline, compared to control/vehicle rats (Fig. 2; two-way ANOVA; factors: stress $\times$ time; $F_{(1,173)}=7.433 ; t=$ 2.726; $P=0.007$, control/vehicle: $n=15$ rats; stress/vehicle: $n=14$ rats). This hyperlocomotion was exacerbated in stress/vehicle animals following MK-801 administration $(t=4.399 ; P<0.001)$. In contrast, there were no differences in locomotor activity at baseline or after MK-801 administration between stressed and control animals treated with Suvorexant (Fig. 2). When comparing total distance traveled after MK-801 administration, across groups, we observed a main effect of stress on locomotor activity (Fig. 2; two-way ANOVA; factors: stress $\times$ drug; $F_{(1,54)}=4.316 ; t=2.077 ; P=0.043, n=13-15$ rats $/$ group). Specifically, stress/vehicle animals exhibited significantly increased locomotor activity, compared to control/vehicle animals (Holm-Sidak: $t=2.747, p=$ 0.014). This hyperlocomotion was reversed in stressed animals treated with $10 \mathrm{mg} / \mathrm{kg}$ of Suvorexant (Holm-Sidak: $t=2.555, p=0.014 ; n=13$ rats). Taken together, these data suggest two-day inescapable footshock stress can exacerbate MK-801 induced hyperlocomotion and that this aberrant behavior can be reversed with Suvorexant. 


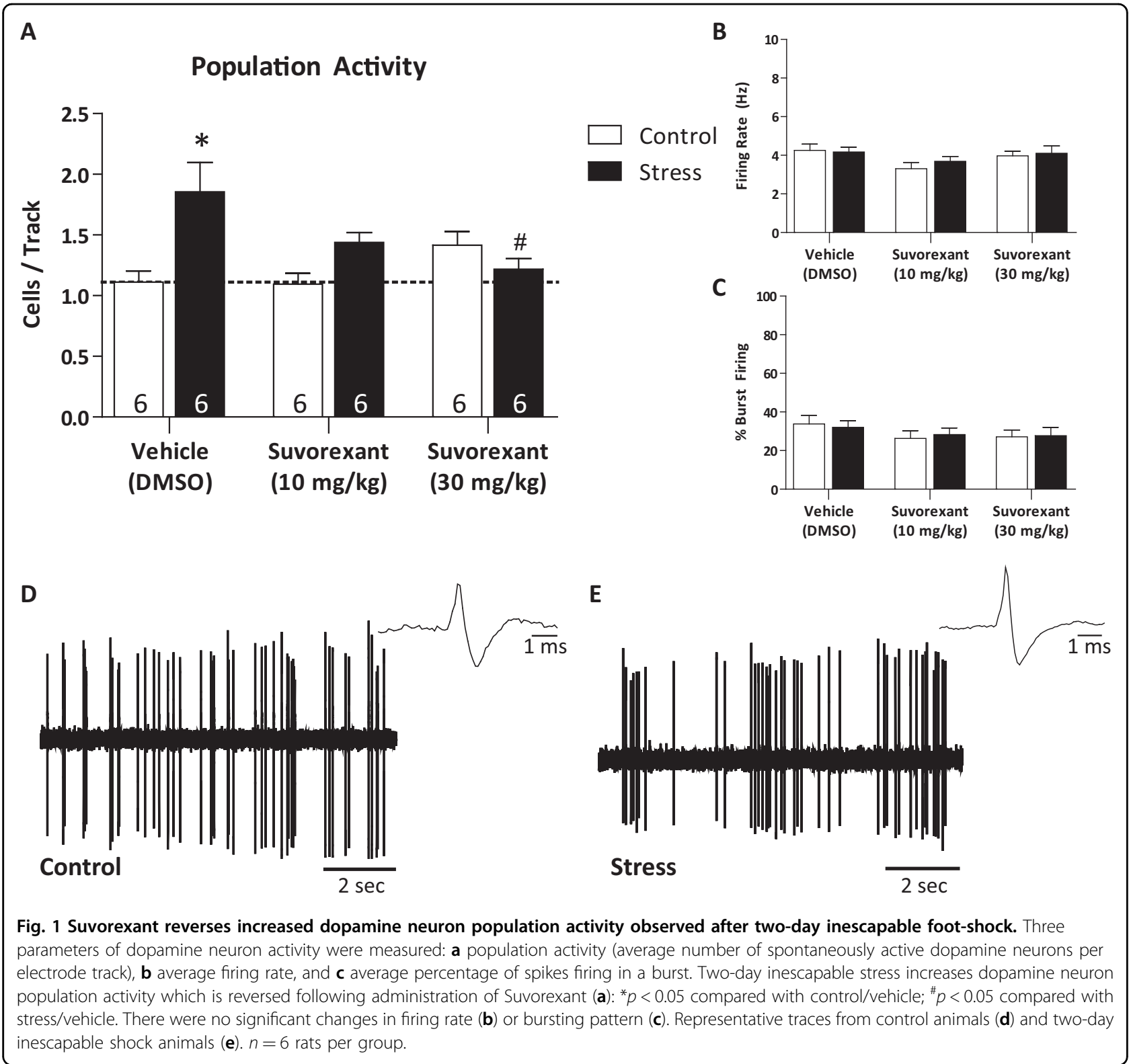

Selective $O X_{1} R$ and $O X_{2} R$ antagonists both reverse aberrant VTA dopamine neuron population activity following stress

After observing that blockade of both $\mathrm{OX}_{1} \mathrm{R}$ and $\mathrm{OX}_{2} \mathrm{R}$, with the DORA Suvorexant, reversed stress-induced deficits in both dopamine neuron population activity and dopamine-dependent behaviors, we investigated the ability of each receptor subtype to reverse behavioral and physiological changes after stress. Similar to our Suvorexant experiments, we found a main effect of stress (Fig. 3; twoway ANOVA; factors: stress $\times$ drug; $F_{(1,41)}=7.130 ; t=$ $2.670 ; p=0.011, n=7$ rats/group). Stress/vehicle animals displayed an increase in the number of spontaneously active dopamine cells per track compared to control/ vehicle animals (Holm-Sidak: $t=5.224, p=<0.001$; control/vehicle: $1.06 \pm 0.04$ cells per track; stress/vehicle; $1.69 \pm 0.13$ cells per track). Interestingly, this effect was attenuated following administration of either an $\mathrm{OX}_{1} \mathrm{R}$ antagonist, SB334867 $(10 \mathrm{mg} / \mathrm{kg})$, or an $\mathrm{OX}_{2} \mathrm{R}$ antagonist, EMPA $(10 \mathrm{mg} / \mathrm{kg})$. SB334867/stress animals displayed a complete reversal of aberrant dopamine neuron activity compared to stress/vehicle animals $(p=<0.001 ; 1.04 \pm 0.09$ cells per track), as did EMPA/stress animals $(p=<0.001$; $1.21 \pm 0.09$ cells per track). Additionally, we examined two other parameters of dopamine cell activity; firing rate and percentage bursting. There were no significant differences in firing rate (control/vehicle: $n=50$ neurons; $4.22 \pm$ $0.26 \mathrm{~Hz}$; stress/vehicle: $n=89$ neurons; $3.95 \pm 0.23 \mathrm{~Hz}$; 


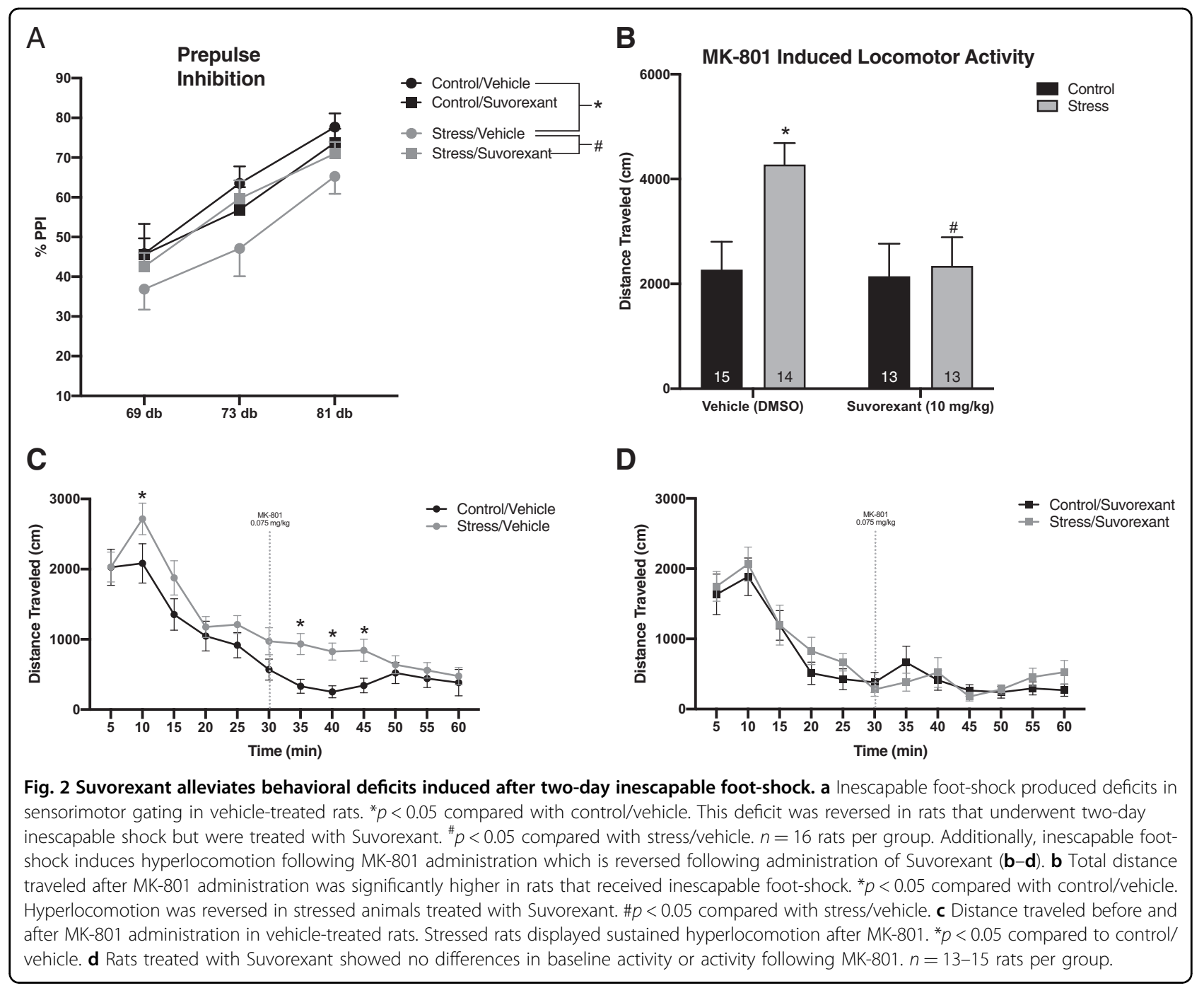

control/SB334867: $\mathrm{n}=53$ neurons; $3.51 \pm 0.25 \mathrm{~Hz}$; stress/ SB334867: $n=49$ neurons; $3.72 \pm 0.25 \mathrm{~Hz}$; control/EMPA: $n=63$ neurons; $4.09 \pm 0.23 \mathrm{~Hz}$; stress/EMPA: $n=57$ neurons; $4.07 \pm 0.31 \mathrm{~Hz}$ ) or percentage bursting (control/vehicle: $n=50$ neurons; $27.35 \pm 3.78 \%$; stress/vehicle: $n=89$ neurons; $24.31 \pm 2.58 \%$; control/SB334867: $n=53$ neurons; $26.85 \pm 3.89 \%$; stress/SB334867: $n=49$ neurons; $21.59 \pm$ $3.34 \%$; control/EMPA: $n=63$ neurons; $26.28 \pm 2.97 \%$; stress/EMPA: $n=57$ neurons; $24.78 \pm 3.24 \%)$. Collectively, these data suggest blockade of either $\mathrm{OX}_{1} \mathrm{R}$ or $\mathrm{OX}_{2} \mathrm{R}$ can alleviate aberrant dopamine cell activity, following stress. However, given the sedative effects of $\mathrm{OX}_{2} \mathrm{R}$ antagonists, selective $\mathrm{OX}_{1} \mathrm{R}$ antagonists may be a more feasible treatment option for PTSD and comorbid psychosis.

\section{$\mathrm{OX}_{1} \mathrm{R}$ antagonist, but not $\mathrm{OX}_{2} \mathrm{R}$ antagonist, reverses stress- induced deficits in PPI}

After demonstrating the ability of both $\mathrm{OX}_{1} \mathrm{R}$ and $\mathrm{OX}_{2} \mathrm{R}$ receptor antagonists to alleviate stress-induced increases in VTA dopamine neuron activity, we examined the ability of the $\mathrm{OX}_{1} \mathrm{R}$ antagonist, SB334867, and the $\mathrm{OX}_{2} \mathrm{R}$ antagonist, EMPA to reverse stress-induced deficits in PPI. Unlike our observations from our in vivo dopamine electrophysiology experiments, we found that only SB334867 was able to reverse deficits in PPI (Fig. 4). As expected, we observed a main effect of pre-pulse intensity (Fig. $4 ; p<0.001 ; n=10$ rats/group). Similar to previous observations, we found a main effect of stress (Fig. 4; three-way ANOVA; factors: stress $\times$ drug $\times \mathrm{dB} ; F_{(1,179)}=$ $7.385 ; t=2.717 ; p=0.007)$. Additionally, we observed a main effect of drug, in which SB334867 treated animals displayed a statistically significant difference in PPI than stress/vehicle animals $(t=3.358, p=0.003, n=10$ animals/group) but EMPA treated animals did not differ statistically from stress/vehicle-treated animals (although a trend was observed). No differences were observed in average startle response in animals treated with SB334867 (Holm-Sidak: $t=0.149, p=0.968)$ or EMPA $(t=0.228$, 


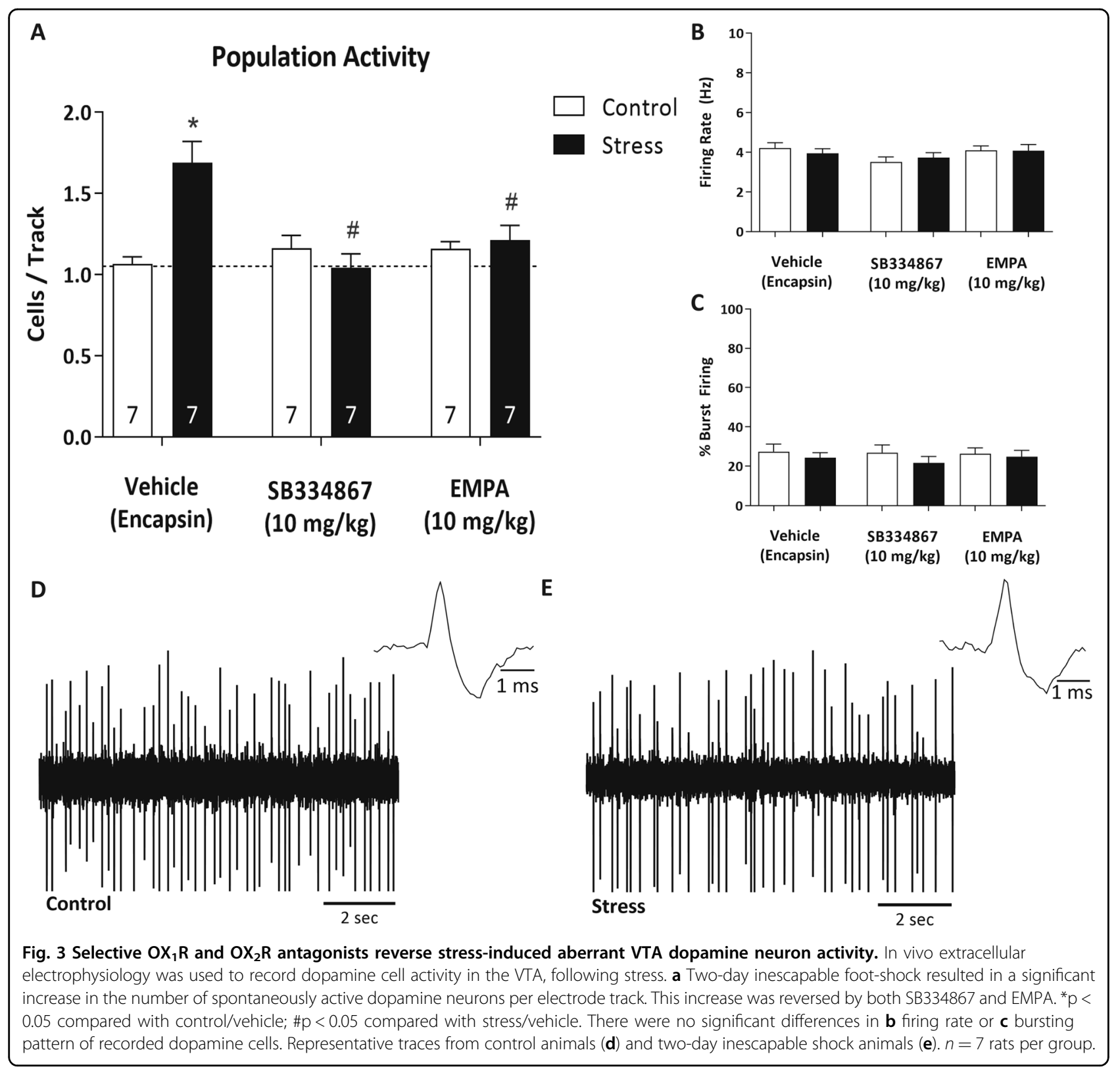

$p=0.994)$ compared to vehicle-treated animals. These data further support the role of $\mathrm{OX}_{1} \mathrm{R}$ antagonists in alleviating symptoms of psychosis in PTSD patients.

\section{Discussion}

In the current experiments, we utilized the FDAapproved DORA, Suvorexant (trade name: Belsomra) ${ }^{45}$, a selective $\mathrm{OX}_{1} \mathrm{R}$ antagonist, $\mathrm{SB} 334867$, and a selective $\mathrm{OX}_{2} \mathrm{R}$ antagonist, EMPA, to demonstrate that stressrelated physiological and behavioral alterations observed in a stress-induced model can be reversed by systemic orexin receptor blockade. In order to model PTSD-like pathophysiology in a rodent, we used the two-day inescapable foot-shock procedure, which has been shown to produce robust behavioral and physiological changes, such as avoidance and anxiety-like behavior, hyperarousal and aggression, and alterations in sleep ${ }^{28-30}$. Here we demonstrate that two-day, inescapable foot-shock produces specific increases in VTA dopamine population activity as well as deficits in dopamine-dependent behaviors (PPI and stimulant-induced hyperlocomotion). Furthermore, systemic administration of either the DORA, Suvorexant, or the selective $\mathrm{OX}_{1} \mathrm{R}$ antagonist, SB334867, completely alleviated stress-induced physiological and behavioral changes. Our results suggest that targeting the orexin system may be a novel therapeutic approach for the treatment of PTSD and comorbid psychosis. 


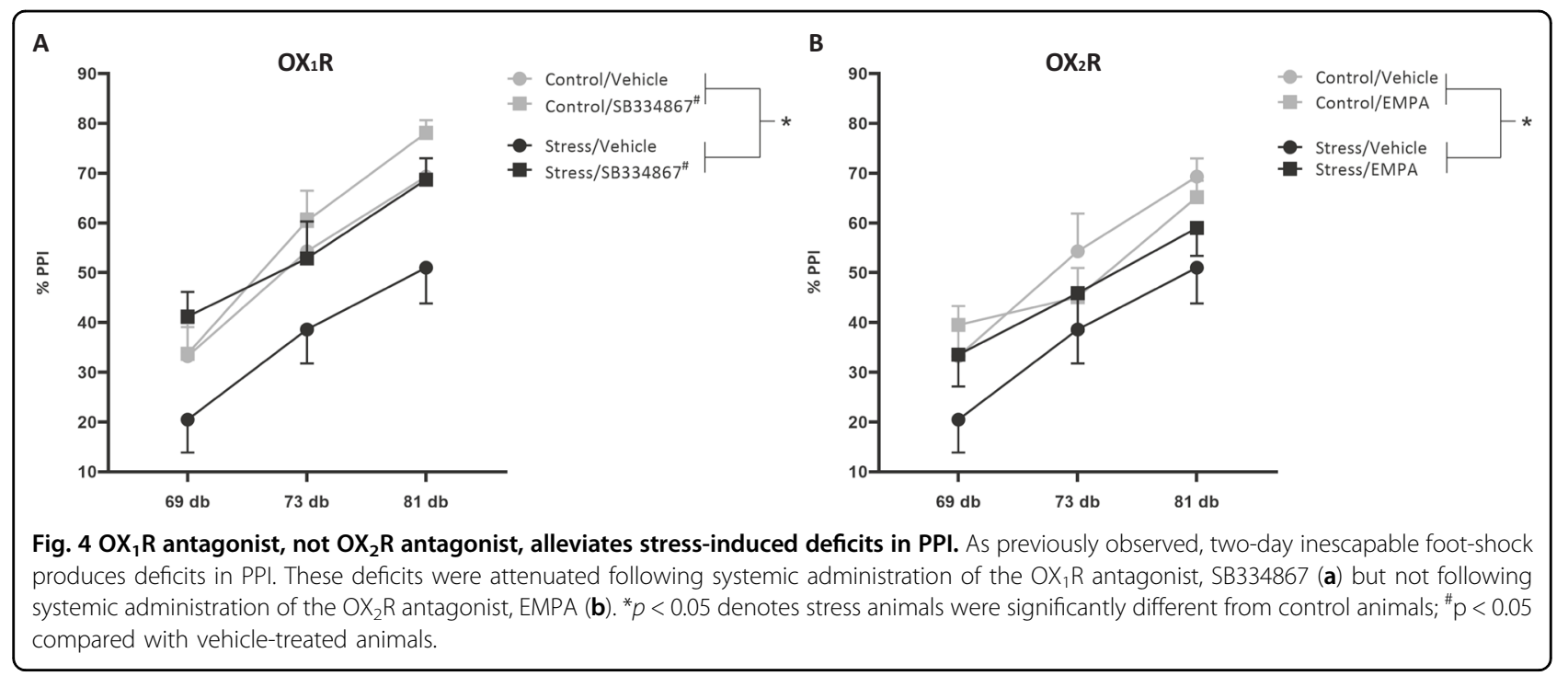

Although the presentation of PTSD and comorbid psychosis is highly heterogeneous, there are multiple brain regions consistently implicated in the disorder, including the thalamus ${ }^{27,46-49}$. The thalamus is composed of multiple nuclei, but the afferent and efferent projections of the PVT position this particular thalamic nucleus to regulate symptoms associated with PTSD and comorbid psychosis. Activation of the PVT has been shown to occur following numerous stressful and aversive events, such as restraint, exposure to predator scent, and footshock $^{50-52}$. Additionally, both structural and metabolic changes within the thalamus are observed in patients with psychotic symptoms ${ }^{53}$. Our laboratory has recently demonstrated that increased glutamatergic transmission from the PVT, specifically, to the NAc causes an increase in the number of spontaneously active dopaminergic neurons in the VTA, through a multisynaptic pathway ${ }^{12}$. Symptoms of psychosis consistently correlate with an increase in mesolimbic dopamine transmission, which can be observed in both humans, using PET imaging' ${ }^{9}$, as well as in animal models of psychosis, using in vivo electrophysiology ${ }^{10}$. While it is difficult to model and measure symptoms of psychosis in a rodent, such as hallucinations and delusions, it is possible to measure changes in dopamine neuron activity. Using in vivo extracellular electrophysiology, we are able to evaluate aspects of dopamine cell activity (population activity, firing rate and burst firing $)^{11}$. We have previously demonstrated, in a rodent model used to study psychosis, that intracranial administration of the DORA, TCS1102, into the PVT can restore dopamine system function, suggesting the PVT may be a novel therapeutic target for the treatment of psychosis ${ }^{27}$. Taken together, data from previous studies suggest the PVT becomes activated following stressful events $^{50-52}$ and that this aberrant activation may contribute to increased dopamine neuron population activity within the VTA that ultimately underlies symptoms of psychosis.

Orexin-containing neurons originate in the lateral hypothalamus and innervate a variety of brain regions, including the PVT ${ }^{14}$. These neurons release orexin neuropeptides, OXA and OXB, which bind to and activate two Goq-protein-coupled receptors, $\mathrm{OX}_{1} \mathrm{R}$ and $\mathrm{OX}_{2} \mathrm{R}^{16}$, which, once activated, increase intracellular calcium concentrations $^{54,55}$. Although orexins were originally discovered for their role in regulating sleep and wakefulness ${ }^{14,22}$, they have now been implicated in host of other behaviors, including avoidance behavior ${ }^{56}$, drug-seeking behavior $^{23,24}$, and psychosis ${ }^{25}$. Of interest to the current project, is the ability of both OXA and OXB to dosedependently modulate PVT neuron activity and the ability of a DORA to reverse aberrant dopamine neuron activity when administered directly in the $\mathrm{PVT}^{26,27}$. Thus, we posit that pharmacological blockade of orexin receptors may inhibit stress-induced activation of the PVT and subsequently restore downstream dopamine system function and associated behaviors in a rodent model of PTSD and comorbid psychosis.

Indeed, we found that systemic administration of the FDA-approved DORA, Suvorexant, was able to reverse both aberrant dopamine neuron population activity and behavioral deficits in PPI and stimulant-induced locomotor activity, following stress. It is important to note that although Suvorexant is currently prescribed as a hypnotic, it does not significantly alter the time taken to fall asleep ${ }^{57}$. Rather, Suvorexant administration provides relief for insomnia by decreasing the time to wake after sleep onset and increasing the total time spent asleep ${ }^{58}$. Our results suggest lower, non-sedative doses of Suvorexant are sufficient to alleviate behavioral correlates of 
psychosis. Indeed, in our study, the administration of Suvorexant $(10 \mathrm{mg} / \mathrm{kg})$ did not significantly reduce baseline locomotor activity (distance traveled), as shown in Fig. 2D, suggesting that this dose does not produce overt sedative effects. Conversely, initial pilot studies demonstrated that the high dose of Suvorexant $(30 \mathrm{mg} / \mathrm{kg})$ dramatically reduced spontaneous locomotor activity (by $>80 \%$ ), prompting us to halt any further behavioral assays using this dose. This is consistent with the literature, in which $30 \mathrm{mg} / \mathrm{kg}$ of Suvorexant has been reported to reduce baseline locomotor activity ${ }^{59}$. Although $30 \mathrm{mg} / \mathrm{kg}$ of Suvorexant resulted in behavioral sedation in our pilot experiment, we did not observe decreases in dopamine neuron population activity in our control group, which could be expected in sedation alone resulted in decreased dopamine neuron activity. Taken together, these data suggest lower, non-sedating doses of Suvorexant may be effective at treating comorbid psychosis.

After demonstrating that antagonizing both $\mathrm{OX}_{1} \mathrm{R}$ and $\mathrm{OX}_{2} \mathrm{R}$ with the DORA, Suvorexant, restores stressinduced increases in VTA dopamine neuron activity, we examined the ability of each orexin receptor subtype to selectively reverse aberrant dopamine neuron activity. Although both orexin receptors play a role in the sleepwake cycle, $\mathrm{OX}_{2} \mathrm{R}$ is necessary for wakefulness, while $\mathrm{OX}_{1} \mathrm{R}$ plays a less critical role ${ }^{60}$. Evidence from both human and animal studies have shown that antagonism or loss of $\mathrm{OX}_{2} \mathrm{R}$, but not $\mathrm{OX}_{1} \mathrm{R}$, results in narcoleptic symptoms ${ }^{61-63}$. Thus, selective antagonism of $\mathrm{OX}_{1} \mathrm{R}$ in the treatment of PTSD and comorbid psychosis would be ideal to avoid sedation while still decreasing aberrant dopamine transmission. We therefore examined the role of each orexin receptor in modulating aberrant dopamine transmission following stress. Given that both orexin receptors are expressed in the PVT and that direct injections of OXA and OXB into the PVT can cause significant increases in dopamine neuron population activity $^{26}$, we posited that selective antagonism of either receptor would reverse stress-induced increases in VTA dopamine neuron activity. After administration of the selective $\mathrm{OX}_{1} \mathrm{R}$ antagonist, SB334867 $(10 \mathrm{mg} / \mathrm{kg})^{64}$, and selective $\mathrm{OX}_{2} \mathrm{R}$ antagonist, EMPA $(10 \mathrm{mg} / \mathrm{kg})^{36}$, we found that both compounds were able to reverse aberrant dopamine system function in our rodent model of PTSD and psychosis. These studies provide further evidence that targeting the orexin receptors in the PVT is useful at treating dopamine dysfunction in a rodent model of PTSD and comorbid psychosis.

Lastly, we evaluated the ability of the selective orexin receptor compounds to reverse a dopamine-dependent behavior associated with psychosis. PPI is a commonly used assay to assess psychosis in humans and that can be effectively measured in rats ${ }^{32}$. Although both SB334867 $\left(\mathrm{OX}_{1} \mathrm{R}\right)$ and EMPA $\left(\mathrm{OX}_{2} \mathrm{R}\right)$ were able to reverse aberrant dopamine neuron population activity, only SB334867 was able to reverse stress-induced deficits in PPI (although a trend was observed with EMPA). While sensorimotor gating is dependent on dopamine release in the NAc, the ability to regulate PPI is not exclusive to this brain region. Brain regions such as the hippocampus, mPFC, and amygdala have also been shown to contribute to deficits in $\mathrm{PPI}^{32}$ and previous studies have demonstrated orexin receptor expression and orexin receptor signaling in these regions is altered following stress $^{65-67}$. Furthermore, the expression of $\mathrm{OX}_{1} \mathrm{R}$ and $\mathrm{OX}_{2} \mathrm{R}$ differs within these regions. Namely, $\mathrm{OX}_{1} \mathrm{R}$ is highly expressed in CA1 and CA2 hippocampal fields, mPFC subregions (ie. the infralimbic cortex), and various amygdala nuclei while $\mathrm{OX}_{2} \mathrm{R}$ shows minimal expression in all these regions ${ }^{18}$, suggesting antagonism of $\mathrm{OX}_{1} \mathrm{R}$ may have direct effects on circuitry responsible for sensorimotor gating. Conversely, dopamine neuron activity in the VTA has been shown to be regulated by the $\mathrm{PVT}^{12,27}$, which is densely innervated by orexin containing neurons and displays substantial expression of both $\mathrm{OX}_{1} \mathrm{R}$ and $\mathrm{OX}_{2} \mathrm{R}$. These differences in expression of orexin receptor subtypes could explain the differential effects of $\mathrm{OX}_{1} \mathrm{R}$ and $\mathrm{OX}_{2} \mathrm{R}$ antagonists on PPI and VTA dopamine neuron activity but further investigation is warranted to fully elucidate this finding.

In conclusion, we have demonstrated that by targeting orexin receptors with the FDA-approved DORA, Suvorexant $(30 \mathrm{mg} / \mathrm{kg}), \mathrm{OX}_{1} \mathrm{R}$ antagonist, SB334867, and the $\mathrm{OX}_{1} \mathrm{R}$ antagonist, EMPA, we are able to restore normal dopamine system function in a rodent model of stressinduced pathophysiology relevant to PTSD and comorbid psychosis. Further, a low, non-sedating dose of the FDAapproved antagonist, Suvorexant, was able to reverse PPI deficits and eliminate increased sensitivity to psychomotor stimulants observed in the stress model. Additionally, the $\mathrm{OX}_{1} \mathrm{R}$ antagonist, SB334867, can also alleviate deficits in PPI observed in our model. Taken together, these findings suggest that pharmacological blockade of orexin receptors, specifically $\mathrm{OX}_{1} \mathrm{R}$, may be a novel therapeutic approach for the treatment of PTSD and comorbid psychosis.

\section{Funding and disclosure}

This work was supported by Merit Award \#BX004693 from the United States Department of Veterans Affairs, Biomedical Laboratory Research and Development Service, and the National Institutes of Health (RO1-MH090067).

\section{Author contributions}

H.B.E. made contributions to the design of the work, acquisition, analysis and interpretation of the data, as well as drafting and editing the manuscript. S.M.P. contributed to acquisition, analysis and interpretation of the data, and revising and editing the manuscript content. J.J.D. contributed to acquisition, analysis and interpretation of the data, and revising and editing the manuscript content. D.J.L. contributed to the concept and design of the study, revising and editing manuscript content, as well as providing final approval of the document. 


\section{Conflict of interest}

The authors declare that they have no conflict of interest.

\section{Publisher's note}

Springer Nature remains neutral with regard to jurisdictional claims in published maps and institutional affiliations.

Received: 19 August 2020 Revised: 8 January 2021 Accepted: 18 January 2021

Published online: 08 February 2021

\section{References}

1. Association, A. P., Diagnostic and Statistical Manual of Mental Disorders. 5th ed. 2013, Washington, DC.

2. Nemeroff, C. B. et al. Posttraumatic stress disorder: a state-of-the-science review. J. Psychiatr. Res. 40, 1-21 (2006).

3. Shalev, A. Y. et al. Prospective study of posttraumatic stress disorder and depression following trauma. Am. J. Psychiatry 155, 630-637 (1998).

4. Mueser, K. T. et al. Trauma and posttraumatic stress disorder in severe mental illness. J. Consult Clin. Psychol. 66, 493-499 (1998).

5. Braakman, M. H., Kortmann, F. A. \& van den Brink, W. Validity of 'post-traumatic stress disorder with secondary psychotic features': a review of the evidence. Acta Psychiatr. Scand. 119, 15-24 (2009).

6. Hardy, K. V. \& Mueser, K. T. Editorial: trauma, psychosis and posttraumatic stress disorder. Front Psychiatry 8, 220 (2017).

7. Leucht, S. et al. Comparative efficacy and tolerability of 15 antipsychotic drugs in schizophrenia: a multiple-treatments meta-analysis. Lancet 382, 951-962 (2013).

8. Laruelle, M. \& Abi-Dargham, A. Dopamine as the wind of the psychotic fire: new evidence from brain imaging studies. J. Psychopharmacol. 13, 358-371 (1999).

9. Howes, O. D. et al. Elevated striatal dopamine function linked to prodromal signs of schizophrenia. Arch. Gen. Psychiatry 66, 13-20 (2009).

10. Lodge, D. J. \& Grace, A. A. Aberrant hippocampal activity underlies the dopamine dysregulation in an animal model of schizophrenia. J. Neurosci. 27, 11424-11430 (2007)

11. Lodge, D. J. \& Grace, A. A. Hippocampal dysregulation of dopamine system function and the pathophysiology of schizophrenia. Trends Pharm. Sci. 32, 507-513 (2011).

12. Perez, S. M. \& Lodge, D. J. Convergent inputs from the hippocampus and thalamus to the nucleus accumbens regulate dopamine neuron activity. J. Neurosci. 38, 10607-10618 (2018).

13. Penzo, M. A. et al. The paraventricular thalamus controls a central amygdala fear circuit. Nature 519, 455-459 (2015).

14. de Lecea, L. et al. The hypocretins: hypothalamus-specific peptides with neuroexcitatory activity. Proc. Natl Acad. Sci. USA 95, 322-327 (1998).

15. Kirouac, G. J. Placing the paraventricular nucleus of the thalamus within the brain circuits that control behavior. Neurosci. Biobehav. Rev. 56, 315-329 (2015).

16. Milbank, E. \& Lopez, M. Orexins/hypocretins: key regulators of energy homeostasis. Front. Endocrinol. 10, 830 (2019).

17. Trivedi, P., Yu, H. \& MacNeil, D. J. Van der Ploeg, L.H., Guan, X.M. Distribution of orexin receptor mRNA in the rat brain. FEBS Lett. 438, 71-75 (1998).

18. Marcus, J. N. et al. Differential expression of orexin receptors 1 and 2 in the rat brain. J. Comp. Neurol. 435, 6-25 (2001).

19. Ferno, J., Senaris, R., Dieguez, C., Tena-Sempere, M. \& Lopez, M. Orexins (hypocretins) and energy balance: more than feeding. Mol. Cell Endocrinol. 418 (Pt 1), 17-26 (2015).

20. Lopez, M., Tena-Sempere, M. \& Dieguez, C. Cross-talk between orexins (hypocretins) and the neuroendocrine axes (hypothalamic-pituitary axes). Front. Neuroendocrinol. 31, 113-127 (2010).

21. Willie, J. T., Chemelli, R. M., Sinton, C. M. \& Yanagisawa, M. To eat or to sleep? Orexin in the regulation of feeding and wakefulness. Annu. Rev. Neurosci. 24, 429-458 (2001).

22. Sakurai, T. et al. Orexins and orexin receptors: a family of hypothalamic neuropeptides and $\mathrm{G}$ protein-coupled receptors that regulate feeding behavior. Cell 92, 573-585 (1998).
23. Mohammadkhani, $\mathrm{A}$. et al. Orexin-1 receptor signaling in ventral pallidum regulates motivation for the opioid remifentanil. J. Neurosci. 39, 9831-9840 (2019).

24. Steiner, N. et al. Hypocretin/orexin deficiency decreases cocaine abuse liability. Neuropharmacology 133, 395-403 (2018).

25. Han, Y., Yuan, K., Zheng, Y. \& Lu, L. Orexin receptor antagonists as emerging treatments for psychiatric disorders. Neurosci. Bull. 36, 432-448 (2019).

26. Ishibashi, M. et al. Effects of orexins/hypocretins on neuronal activity in the paraventricular nucleus of the thalamus in rats in vitro. Peptides 26, 471-481 (2005).

27. Perez, S. M. and Lodge, D. J. Orexin modulation of VTA dopamine neuron activity: relevance to schizophrenia (Manuscript submitted for publication). 2020.

28. Van Dijken, H. H., Van der Heyden, J. A., Mos, J. \& Tilders, F. J. Inescapable footshocks induce progressive and long-lasting behavioural changes in male rats. Physiol. Behav. 51, 787-794 (1992).

29. Pawlyk, A. C., Jha, S. K., Brennan, F. X., Morrison, A. R. \& Ross, R. J. A rodent model of sleep disturbances in posttraumatic stress disorder: the role of context after fear conditioning. Biol. Psychiatry 57, 268-277 (2005).

30. Pynoos, R. S., Ritzmann, R. F., Steinberg, A. M., Goenjian, A. \& Prisecaru, I. A behavioral animal model of posttraumatic stress disorder featuring repeated exposure to situational reminders. Biol. Psychiatry 39, 129-134 (1996).

31. Braff, D. L. \& Geyer, M. A. Sensorimotor gating and schizophrenia. Hum. Anim. model Stud. Arch. Gen. Psychiatry 47, 181-188 (1990).

32. Swerdlow, N. R., Geyer, M. A. \& Braff, D. L. Neural circuit regulation of prepulse inhibition of startle in the rat: current knowledge and future challenges. Psychopharmacology 156, 194-215 (2001).

33. Lieberman, J. A., Kane, J. M. \& Alvir, J. Provocative tests with psychostimulant drugs in schizophrenia. Psychopharmacology 91, 415-433 (1987).

34. Born, S., Gauvin, D. V., Mukherjee, S. \& Briscoe, R. Preclinical assessment of the abuse potential of the orexin receptor antagonist, suvorexant. Regul. Toxicol. Pharm. 86, 181-192 (2017).

35. Langmead, C. J. et al. Characterisation of the binding of [3H]-SB-674042, a novel nonpeptide antagonist, to the human orexin-1 receptor. Br. J. Pharm. 141, 340-346 (2004).

36. Malherbe, P. et al. Biochemical and behavioural characterization of EMPA, a novel high-affinity, selective antagonist for the OX(2) receptor. Br. J. Pharm. 156, 1326-1341 (2009).

37. Hyland, B. I., Reynolds, J. N., Hay, J., Perk, C. G. \& Miller, R. Firing modes of midbrain dopamine cells in the freely moving rat. Neuroscience $\mathbf{1 1 4}, 475-492$ (2002).

38. Ungless, M. A. \& Grace, A. A. Are you or aren't you? Challenges associated with physiologically identifying dopamine neurons. Trends Neurosci. 35, 422-430 (2012).

39. Grace, A. A. \& Bunney, B. S. Intracellular and extracellular electrophysiology of nigral dopaminergic neurons-1. Identification and characterization. Neuroscience 10, 301-315 (1983).

40. Paxinos, G., Watson, C. The rat brain in stereotaxic coordinates. (Academic Press: San Diego, 1998).

41. Perez, S. M. \& Lodge, D. J. Hippocampal interneuron transplants reverse aberrant dopamine system function and behavior in a rodent model of schizophrenia. Mol. Psychiatry 18, 1193-1198 (2013).

42. Perez, S. M., Donegan, J. J. \& Lodge, D. J. Effect of estrous cycle on schizophrenia-like behaviors in MAM exposed rats. Behav. Brain Res. 362, 258-265 (2019).

43. Chalkiadaki, K. et al. Development of the MAM model of schizophrenia in mice: Sex similarities and differences of hippocampal and prefrontal cortical function. Neuropharmacology 144, 193-207 (2019).

44. Katz, R. J., Roth, K. A. \& Carroll, B. J. Acute and chronic stress effects on open field activity in the rat: implications for a model of depression. Neurosci. Biobehav. Rev. 5, 247-251 (1981).

45. Cox, C. D. et al. Discovery of the dual orexin receptor antagonist [(7R)-4-(5chloro-1,3-benzoxazol-2-yl)-7-methyl-1,4-diazepan-1-yl][5-methyl-2-(2H -1,2,3triazol-2-yl)phenyl]methanone (MK-4305) for the treatment of insomnia. J. Med. Chem. 53, 5320-5332 (2010).

46. Lanius, R. A. et al. Recall of emotional states in posttraumatic stress disorder: an fMRI investigation. Biol. Psychiatry 53, 204-210 (2003).

47. Tye, K. M. et al. Dopamine neurons modulate neural encoding and expression of depression-related behaviour. Nature 493, 537-541 (2013).

48. Tsai, H. C. et al. Phasic firing in dopaminergic neurons is sufficient for behavioral conditioning. Science 324, 1080-1084 (2009). 
49. Oke, A. F. \& Adams, R. N. Elevated thalamic dopamine: possible link to sensory dysfunctions in schizophrenia. Schizophr. Bull. 13, 589-604 (1987).

50. Bhatnagar, S. \& Dallman, M. Neuroanatomical basis for facilitation of hypothalamic-pituitary-adrenal responses to a novel stressor after chronic stress. Neuroscience 84, 1025-1039 (1998).

51. Baisley, S. K., Cloninger, C. L. \& Bakshi, V. P. Fos expression following regimens of predator stress versus footshock that differentially affect prepulse inhibition in rats. Physiol. Behav. 104, 796-803 (2011)

52. Bubser, M. \& Deutch, A. Y. Stress induces Fos expression in neurons of the thalamic paraventricular nucleus that innervate limbic forebrain sites. Synapse 32, 13-22 (1999).

53. Clinton, S. M. \& Meador-Woodruff, J. H. Thalamic dysfunction in schizophrenia: neurochemical, neuropathological, and in vivo imaging abnormalities. Schizophr. Res 69, 237-253 (2004).

54. Lund, P. E. et al. The orexin OX1 receptor activates a novel Ca2+ influx pathway necessary for coupling to phospholipase C. J. Biol. Chem. 275, 30806-30812 (2000)

55. Holmqvist, T., Akerman, K. E. \& Kukkonen, J. P. Orexin signaling in recombinant neuron-like cells. FEBS Lett. 526, 11-14 (2002).

56. Fraser, K. M. \& Janak, P. H. Stressing the other paraventricular nucleus. Nat. Neurosci. 21, 901-902 (2018).

57. Rhyne, D. N. \& Anderson, S. L. Suvorexant in insomnia: efficacy, safety and place in therapy. Ther. Adv. Drug Saf. 6, 189-195 (2015).

58. Herring, W. J. et al. Orexin receptor antagonism for treatment of insomnia: a randomized clinical trial of suvorexant. Neurology 79, 2265-2274 (2012).
59. Winrow, C. J. et al. Promotion of sleep by suvorexant-a novel dual orexin receptor antagonist. J. Neurogenet. 25, 52-61 (2011).

60. Kalogiannis, M. et al. Cholinergic modulation of narcoleptic attacks in double orexin receptor knockout mice. PLoS ONE 6, e18697 (2011).

61. Beuckmann, C. T. et al. Expression of a poly-glutamine-ataxin-3 transgene in orexin neurons induces narcolepsy-cataplexy in the rat. J. Neurosci. 24 4469-4477 (2004)

62. Scammell, T. E. \& Winrow, C. J. Orexin receptors: pharmacology and therapeutic opportunities. Annu. Rev. Pharm. Toxicol. 51, 243-266 (2011).

63. Thannickal, T. C. et al. Reduced number of hypocretin neurons in human narcolepsy. Neuron 27, 469-474 (2000).

64. Rodgers, R. J. et al. SB-334867, a selective orexin-1 receptor antagonist, enhances behavioural satiety and blocks the hyperphagic effect of orexin-A in rats. Eur. J. Neurosci. 13, 1444-1452 (2001)

65. Han, D., Han, F., Shi, Y., Zheng, S. \& Wen, L. Mechanisms of memory impairment induced by orexin-A via orexin 1 and orexin 2 receptors in post-traumatic stress disorder rats. Neuroscience 432, 126-136 (2020)

66. Liu, R. J. \& Aghajanian, G. K. Stress blunts serotonin- and hypocretin-evoked EPSCs in prefrontal cortex: role of corticosterone-mediated apical dendritic atrophy. Proc. Natl Acad. Sci. USA 105, 359-364 (2008).

67. Salehabadi, S., Abrari, K., Elahdadi Salmani, M., Nasiri, M. \& Lashkarbolouki, T. Investigating the role of the amygdala orexin receptor 1 in memory acquisition and extinction in a rat model of PTSD. Behav. Brain Res. 384, 112455 (2020). 